\title{
VORWORT
}

\section{DES HERAUSGEBERS ZUR DEUTSCHEN AUSGABE}

Das Buch von A. S. PEtrow über ,Enssterv-Räume“ ist die erste umfassende Monographie, in der die durch die ErNsTEINschen Gravitationsgleichungen bestimmten vier dimensionalen RIEMANNschen Mannigfaltigkeiten von der LORENTZMrskowsKIschen Signatur von geometrischen Gesichtspunkten aus behandelt werden. Der Schwerpunkt des Buches sind die Kapitel III-V, die zu einem großen Teil über invariante Klassifizierungen der EnNsTEnNschen Räume zu einem großen Teil auf die Forschungen des Verfassers zurückgehen. Die dabei gewonnenen Gesichtspunkte haben sich von großer Bedeutung für die Êntwicklung der allgemeinen Relativitätstheorie und insbesondere für die Erforschung der mathematischen Figenschaften der Gravitationswellen erwiesen. Dementsprechend sind auch in den letzten Jahren eine größere Anzahl von Arbeiten erschienen, die im engen Zusammenhang mit dem Gegenstand dès Buches stehen bzw. die hier geschilderten Resultate weiterführen.

Professor PETrow hat selbst für die deutsche Auflage die russische Originalausgabe neu durchgesehen und an verschiedenen Stellen ergänzt. - Als Herausgeber der deutschen Ausgabe habe ich verschiedentlich Noten beigetragen und in einem Anhang eine kurze Ubersicht über die Entwicklung des Problems der invariierten Klassifizierung der EiNSTEnsschen Gravitationsfelder seit dem Erscheinen der russischen Ausgabe des Buches „Ernstern-Räume“ (Moskau 1961) gegeben. Ferner ist das Literaturverzeichnis durch weitere Arbeiten ergänzt worden und umfaßt jetzt über 500 Nummern, so daß wohl der größte Teil aller einschlägigen Arbeiten erfaßt ist.

Meinen Mitarbeitern, den Herren Dipl.-Phys. U. KasPer, Dipl.-Phys. E. KREISEL und Dipl.-Phys. E. LIEBschrar, danke ich für ihre Hilfe bei der Durchsicht der Korrekturen. 
